\title{
BW and SS based Handover Analysis of Four States of Mobile Node in a Five Node Network Model
}

\author{
Suresh R. Halhalli \\ Department of EE \\ M.S. Bidve Engineering College \\ Latur (MS), India
}

\author{
Subhash Kulkarni \\ Department of ECE \\ PES Institute of Technology, \\ SC Bangalore (KS), India
}

\author{
K. S. R. Anjaneyulu \\ Department of EEE \\ JNTUA College of Engineering \\ Anantapuramu (AP), India
}

\begin{abstract}
Handover algorithms based on different performance metric are used to provide seamless handover and need to be evaluated in terms of unnecessary handover and missing handovers. Wrong decision probability (WDP) is one such performance metric used to measure the efficiency of handover algorithms and is measured based on Unnecessary handover probability (UHP) and Missing handover probability (MHP) for handover evaluation. In this work handover probability (HP), UHP, MHP and WDP are computed for a five node network model. The handoff algorithm for the five node network model is designed based on combination of Received signal strength (RSS) and Bandwidth (BW), and is evaluated using Wrong Decision Probability model considering the four states of mobile node, namely Cooperative state, failed state, selfish state and malicious state. Analytical and simulation results are presented to validate the vertical handover. Results are compared with the results of single state five node network model.
\end{abstract}

\section{Keywords}

Missing handover probability (MHP), Unnecessary handover probability (UHP), Wrong decision probability (WDP), Bandwidth (BW), Received Signal strength (RSS),

\section{INTRODUCTION}

Next generation wireless networks are expected to support integration of divergent access network technologies such as vehicular ad hoc networks (VANETs), Wireless Wide Area Networks (WWANs), Wireless Local Area Networks (WLANs), Wireless Personal Area Networks (WPANs), Wireless Metropolitan Area Networks (WMANs), Universal Mobile Telecommunications Systems (UMTSs), mobile adhoc networks (MANETs) and regional/global area networks such as terrestrial broadcasting, satellite communication etc. [1]. Such heterogeneous networks support a different set of specific services and will coexist to offer services ranging from low data rate, non real time applications to high speed real time, multimedia applications, providing enough bandwidth, coverage area, data security for mobile users. Handover need to take place smoothly, when a mobile user moves across the network boundaries or when the user needs are to be satisfied as per the desired performance criteria. Always Best Connected (ABC), anytime, anywhere, and seamless communication are the main features of $4 \mathrm{G}$ technology with heterogeneous all-IP networks [2]. The improvements in the coverage area, data transfer rate, latency, data transfer cost etc. is the main focus of $4 \mathrm{G}$ technology. Seamless mobility i.e. a flawless and fluent handoff scheme that supports the roaming of mobile devices from one wireless system to another is the challenging task of the $4 \mathrm{G}$ heterogeneous all-IP networks.

The main challenge which need to be focused in the $4 \mathrm{G}$ wireless networks is to integrate heterogeneous networks and manage seamless vertical handover (VHO) to select the best network when choice is available or when network boundaries are crossed [3-6]. Handover need to take place when there is deterioration in the received radio signal, the access point capacity for connecting new calls is used up, the co-channel interference, user behavior change, and better choice is available or because of near far effects. Efficient VHO schemes and algorithms need to be developed for seamless mobility between heterogeneous wireless access networks.

The parameters such as Bandwidth, Received signal strength (RSS), Signal to Noise Ratio (SNR), Bit Error Rate (BER), network latency, security, processing Power, velocity, user preference, battery power, cost, throughput, network load balancing, etc. can be considered for designing the vertical handover algorithms [7]. There is no single access technology that fulfills the different needs of the user offering low cost, high-speed, nearly universal coverage, and a high Quality of service, all at the same time [8]. Thus, these algorithms can be broadly classified based on RSS, BW, latency, cost function, etc. and also combination of them and are considered for performing handover [9]. Usage scenarios like number of handovers, handover delays, number of missing handovers (MHO) due to incorrect decisions etc. can be considered for the analysis of these handover algorithms [10]. The handover algorithms can also be evaluated using WDP as a performance metric for measuring the accuracy and efficiency of a handover algorithm for its validation [11].

In [12], WDP is evaluated using network parameters, such as instantaneous estimated throughput, to validate the VHO assessment. In [13] authors concluded that unnecessary handover (UHO) leads to ping-pong effect and brings in low network throughput, longer handoff delay and high dropping probability. In [14], authors used WDP model to evaluate the VHO decision, based on BW as performance metric using two network model. WDP was calculated based on UHP and MHP metric. In [15], WDP, UHP and MHP models are used to predict the probabilities for different decision times and large BW channels in two node network model. In [16], WDP model considering BW as performance metric has been used to predict the probabilities of MHO and UHO for different decision times and bandwidths using three node network model. In [17] the performance of BW plus signal strength based handover algorithms for wrong decisions were studied for three network model. The simulated results show that considering both signal strength and network BW, further reduces number of wrong decisions. In [18] WDP modeling for five network model in cooperative state, considering BW as performance metric has been used to predict the probabilities of MHO and UHO for different decision times and different bandwidths for performing handover leading to an increase in the throughput. In [19] combination of BW and RSS are considered as performance metric for five node network model in cooperative state to predict the probabilities of MHO and UHO using WDP model. There is significant 
improvement in the reduction of wrong decision making, when two network models is replaced by a three network model and then by five network model. Also the Ping-Pong effect caused due to unnecessary handoff has been reduced. However in practice, all the mobile nodes in the network are not cooperative, but can be in other states like failed state, selfish state or malicious state [20-21]. In [22], a five node network model is developed to consider all the four states, namely, cooperative state, failed state, selfish state and malicious state in calculating the handover probability, UHP, MHP and WDP considering BW as performance metric. It was observed that UHP is less by at least $50 \%$ and MHP is less by at least $70 \%$ and so is the case with WDP compared to single state i.e. cooperative state, five node network model.

In this work, a five node network model is developed to consider all the four states, namely, cooperative state, failed state, selfish state and malicious state in calculating the HP, UHP, MHP and WDP considering BW plus RSS as performance metric. Next section presents the analytical models for the UHP, MHP, HP and WDP. Section-3 describes the general algorithms used and section-4 explains the simulated results based on MATLAB coding. Finally important conclusions are drawn in section-5.

\section{ANALYTICAL MODEL}

In this work multiple states Markov model is used to depict the process of mobile terminals (MTs) switching between different networks. These mobile nodes can be in any of the four possible states, namely, Cooperative, Malicious, Selfish or Failed State. Definitions for these four states can be found in ref. [20-22]. The five node network model proposed by [14, $18]$ has all the nodes in cooperative state and is referred to as single state model and is referred to as four state model in [2022], considering the other three states.

\subsection{Single State Model:}

A network having five nodes $\mathrm{n}_{1}, \mathrm{n}_{2}, \mathrm{n}_{3}, \mathrm{n}_{4}$, and $\mathrm{n}_{5}$ in cooperative is referred as single state model and the available bandwidth for the five networks are $\mathrm{B}_{1}, \mathrm{~B}_{2}, \mathrm{~B}_{3}, \mathrm{~B}_{4}$, and $\mathrm{B}_{5}$ respectively.

Let

$$
\begin{aligned}
P_{n_{j} / n_{i}}: & \text { The probability of mobile node moving from } \\
& \text { node } n_{i} \text { to } n_{j} . \\
P_{n_{i} / n_{i}}: & \text { The probability of mobile node continues to } \\
& \text { stay in } n_{i} \text { after a time interval } D
\end{aligned}
$$

Where,

$$
P_{n i / n i}=1-\left[\sum_{\substack{j=1 \\ j \neq i}}^{5} P_{n j / n i}\right]
$$

(1)

For a single state five node network model, the probabilities of a mobile node that continues to stay in the network node $\mathrm{n}_{1}$, $\mathrm{n}_{2}, \mathrm{n}_{3}, \mathrm{n}_{4}$, and $\mathrm{n}_{5}$ can be found in ref. [18].

\subsection{Four State Model:}

Fig. 1 shows a five node network model and the corresponding states in which each mobile node can stay. The four states in which a mobile node can stay are cooperative state, failed state, selfish state or malicious state. A mobile node can move from any of the state to any other state, except that, if a mobile node moves to malicious state, it will never return back to the cooperative state. A mobile node that is in cooperative state in network $n_{1}$ can move to any of the networks $\mathrm{n}_{2}, \mathrm{n}_{3}, \mathrm{n}_{4}$, or $\mathrm{n}_{5}$ and also vice versa is also possible. The probabilities that a mobile node continues to stays in cooperative state of respective networks 1 to 5 can be referred from ref. [22].

As the mobile node can stay in any of the states, it has to move to cooperative state for seamless service. The probability of a mobile node present in cooperative state determines the successful handover probabilities as well. The probabilities of a mobile node moving from failed state, selfish state or malicious state into cooperative state can be referred from the ref. [22].

In this work, these probabilities are used to determine the hand over probabilities. Wrong decision probability as defined in ref. [14, 18, 19 and 22] is;

$$
\mathrm{WDP}=\mathrm{UHP}+\mathrm{MHP}
$$

\section{BW \& SS BASED HANDOVER ALGORITHM}

For a mobile node, the decision to move from one network to another network will be made if the available BW and the RSS in the candidate network is greater than by a threshold value ' $\mathrm{L}$ ', over the available BW and the RSS of the current network. The threshold value can be set to either zero or a positive integer.

Steps involved in the designed algorithm are as follows [19]:

1. Assume that a mobile node is in the network $\mathrm{n}_{1}$ and wants to move to another network, amongst the other available networks $\mathrm{n}_{2}, \mathrm{n}_{3}, \mathrm{n}_{4}$ and $\mathrm{n}_{5}$, which serve the area where the user wants to move to.

2. Define the threshold value of BW as L and RSS as 0 .

3. If the mobile node is at $n_{1}$, then decision is made to switch over to $n_{2}$ when $b_{2}-b_{1} \geq L$ and $s_{2}-s_{1} \geq 0$

4. Else verify $b_{3}-b_{1} \geq L$ and $s_{3}-s_{1} \geq 0$ then switch over to $n_{3}$ if $\mathrm{b}_{3}-\mathrm{b}_{1} \geq \mathrm{L}$ and $\mathrm{s}_{3}-\mathrm{s}_{1} \geq 0$ is true.

5. Else verify $b_{4}-b_{1} \geq L$ and $s_{4}-s_{1} \geq 0$ then switch over to $n_{4}$ if $\mathrm{b}_{4}-\mathrm{b}_{1} \geq \mathrm{L}$ and $\mathrm{s}_{4}-\mathrm{s}_{1} \geq 0$ is true.

6. Else verify $b_{5}-b_{1} \geq L$ and $s_{5}-s_{1} \geq 0$ then switch over to $n_{5}$ if $\mathrm{b}_{5}-\mathrm{b}_{1} \geq \mathrm{L}$ and $\mathrm{s}_{5}-\mathrm{s}_{1} \geq 0$ is true.

7. Else maintain the status quo.

In the above algorithm, $\mathrm{s}_{1}, \mathrm{~s}_{2}, \mathrm{~s}_{3}, \mathrm{~s}_{4}$ and $\mathrm{s}_{5}$ represent the normalized SNR of the received signal. The normalized SNR is the ratio of SNR of the signal and SNR of the peak or reference signal.

The BWs of the networks are assumed to be static instead of dynamic which may be the case practically for the convenience of simulation.

Hence,

$$
\operatorname{P}_{\mathbf{n i} / \mathbf{n j}}=\operatorname{Pr}\{b i-b j \geq L\} \text { and } \operatorname{Pr}\{s i-s j \geq 0\}
$$

Where, $b_{i}$ and $b_{j}$ are the available BW of the network for $i=1,2$, $3,4,5$ and for $\mathrm{j}=1,2,3,4,5$. $\mathrm{P}_{\mathrm{ni} / \mathrm{nj}}$ is the probabilities of the networks for $i=1,2,3,4,5$ and for $j=1,2,3,4,5$. $L$ is the predefined threshold value of BW. $s_{i}$ and $s_{j}$ are the signal strength of the network for $i=1,2,3,4,5$ and for $j=1,2,3,4,5$.

The probability of occupied BW and amplitude of signal strength can be referred from [18]. 


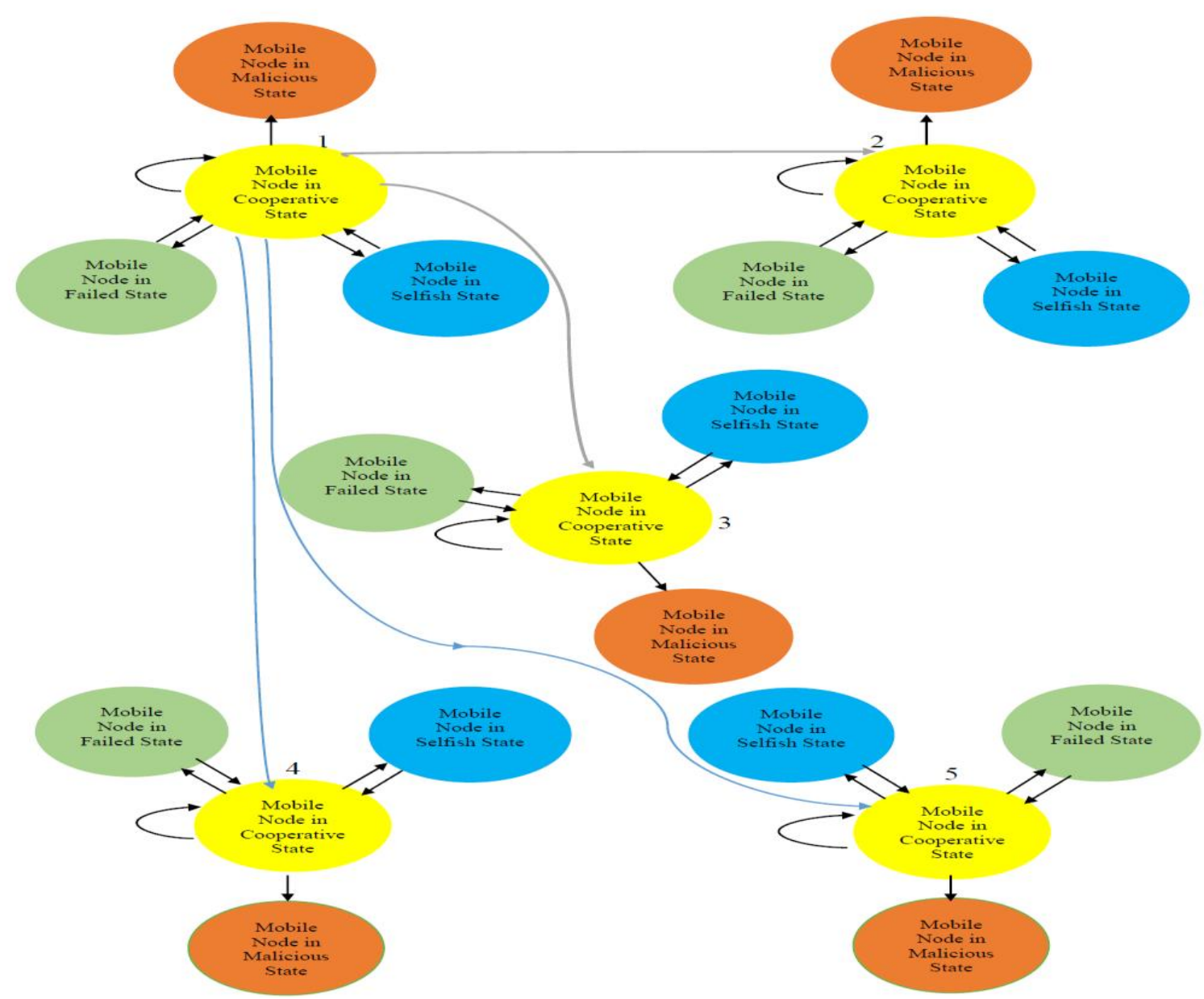

Fig 1: Markov State Five Node Network Model

Following are the parameters used for simulation;

\section{- Site related parameters:}

$>$ Number of networks $=5$.

$>$ Number of Clusters in the system $=1$

- Bandwidth calculations related parameters:

$>$ Initial traffic intensity $=1$ user.

$>$ Assumed number of channels per network $=20$.

$>$ Number of movements for each user from the current

network $=5$

$>$ Threshold, $\mathrm{L}$ is a parameter, default $=0$

- Signal strength calculations related parameters:

$>$ Amplitude of the signal $=1$

$>$ Signal variation is calculated over 500 seconds

$>$ Probability of signal strength in candidate network being higher than, in current network, is 0.5

For a five node network model the Handover probability, UHP, MHP and WDP expressions can be referred from ref. [18-19].

\section{SIMULATION RESULTS}

Bandwidths are varied from 1 to a maximum of 20 on all the five network nodes and simulation are run on the above model. The model was coded in MATLAB. The decision time is also varied for $\mathrm{D}=11 \mathrm{~ms}$ to $15 \mathrm{~ms}$. Since the decision time is varied, the probabilities of mobile node moving from cooperative state to failed state, selfish state or malicious state and vice versa also varies. In this model following simulation factors similar to [22] are used.

- Lift Time, $T_{\text {Life-m }} \quad=\mathrm{D}$

- Residence time, $T_{\text {Residence- } m}=\mathrm{D} / 4$

- Attack time, $T_{\text {attack-m }}=\mathrm{D} / 4$

- Recovery time, $T_{\operatorname{Re} c-m}=\mathrm{D} / 4$

- Selfish time, $T_{\text {Selfish }-m}=\mathrm{D} / 4$

- Probability of attack, $\mathrm{q}_{\mathrm{a}} \quad=0.3$

- $\frac{T C_{T h r-m}}{T C_{M a x-m}}=0.01$

- $\frac{k_{a}}{N} \quad=0.01$

Fig. 2 and 3 shows the UHP's versus bandwidth (traffic density) for single state and four state model for different decision times, $\mathrm{D}=11$ and $13 \mathrm{~ms}$, considering BW plus RSS as performance metric. As the handover probabilities depend on the decision time, it can be noticed that the UHP increases with increase in decision time from 11 to $13 \mathrm{~ms}$ and increase in traffic density. Also it is observed that there is significant improvement in UHP when single state model is replaced by four state model. 


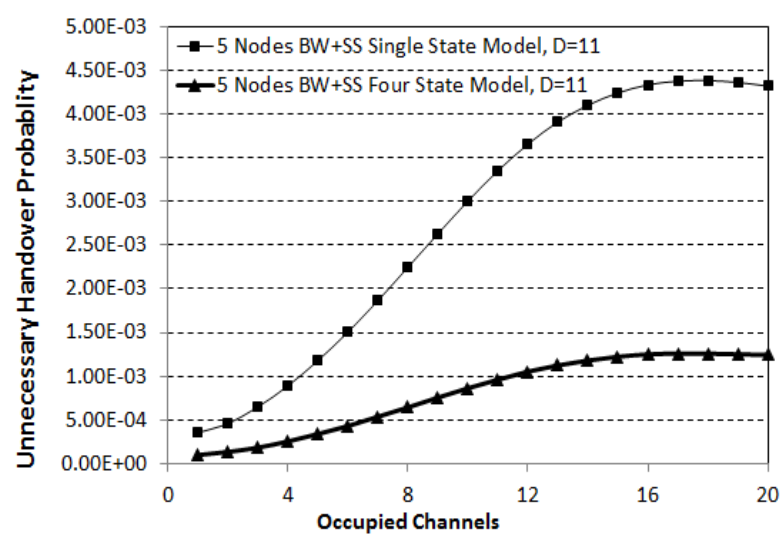

Fig. 2: Unnecessary Handover Probability versus Traffic Density for BW plus RSS based SingleState and Four State Models with $\mathrm{D}=11 \mathrm{~ms}$.

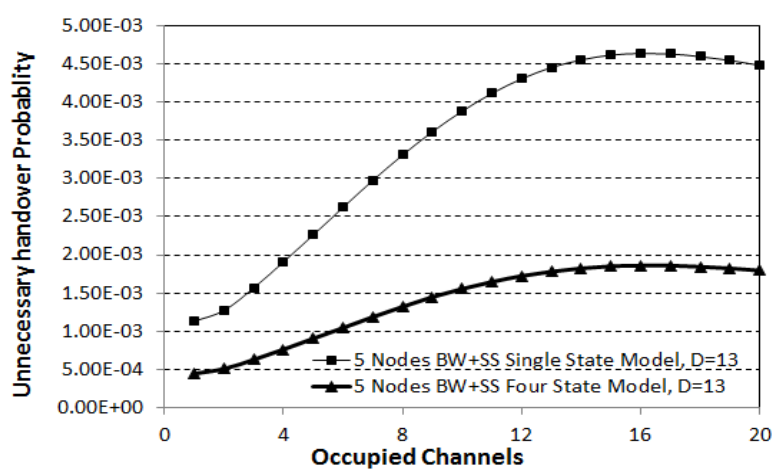

Fig. 3: Unnecessary Handover Probability versus Traffic Density for BW plus RSS based SingleState and Four State Models with $\mathrm{D}=\mathbf{1 3} \mathrm{ms}$.

Fig. 4 and 5 shows the MHP's versus bandwidth (traffic density) for single state four state model for different decision times, D = 12 and $14 \mathrm{~ms}$, considering BW plus RSS as performance metric. As the handover probabilities depend on the decision time, it can be noticed that the MHP increases with increase in decision time from 12 to $14 \mathrm{~ms}$ and increase in traffic density. Also it is observed that there is significant improvement in MHP when single state model is replaced by four state model.

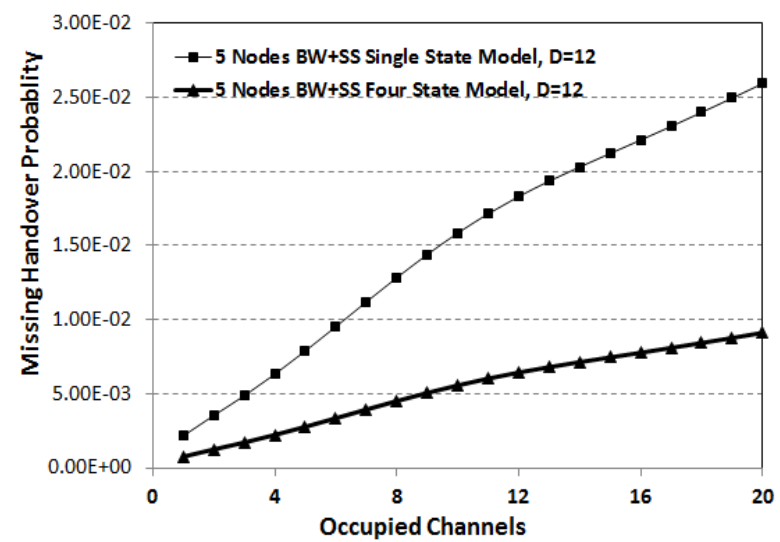

Fig. 4: Missing Handover Probability versus Traffic Density for BW plus RSS based SingleState and Four State Models with $\mathrm{D}=12 \mathrm{~ms}$.

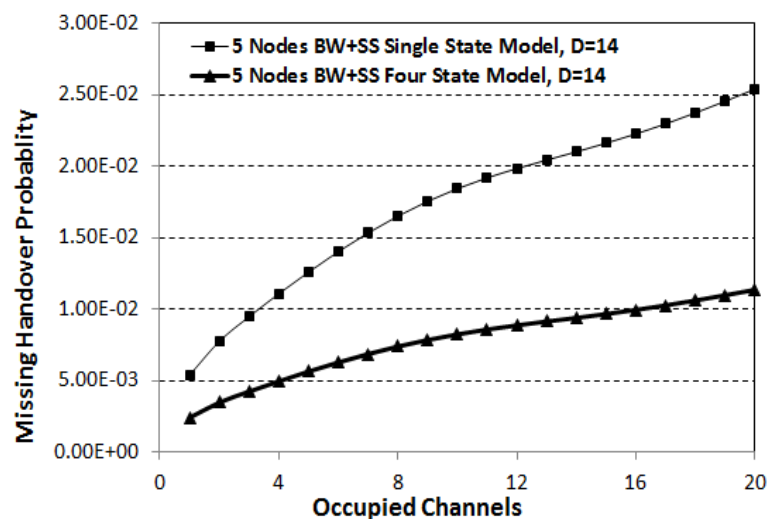

Fig. 5: Missing Handover Probability versus Traffic Density for BW plus RSS based SingleState and Four State Models with $D=14$ ms.

Fig. 6 and 7 shows the WDP's versus bandwidth (traffic density) for single state four state model for different decision times, $\mathrm{D}=11$ and $15 \mathrm{~ms}$, considering $\mathrm{BW}$ plus RSS as performance metric. It can be noticed that the WDP increases with increase in decision time from 11 to $15 \mathrm{~ms}$ and increase in traffic density. Also it is observed that there is significant improvement in WDP when single state model is replaced by four state model.

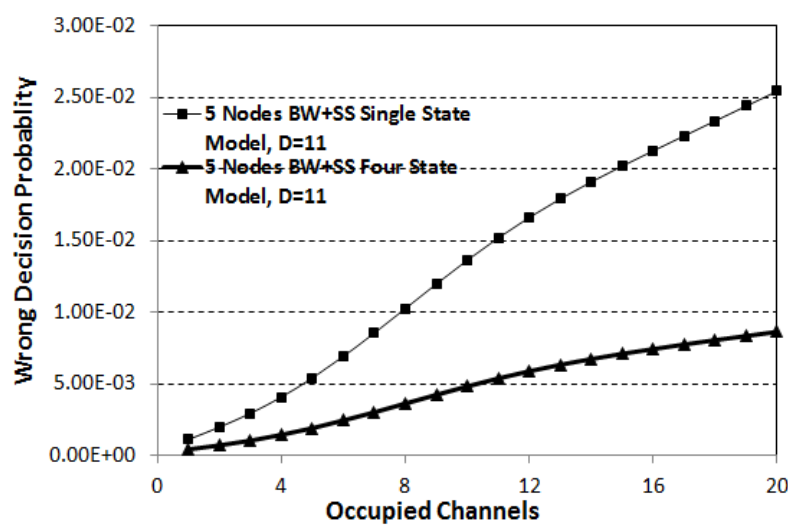

Fig. 6: Wrong Decision Probability versus Traffic Density for BW plus RSS based SingleState and Four State Models with $D=11 \mathrm{~ms}$.

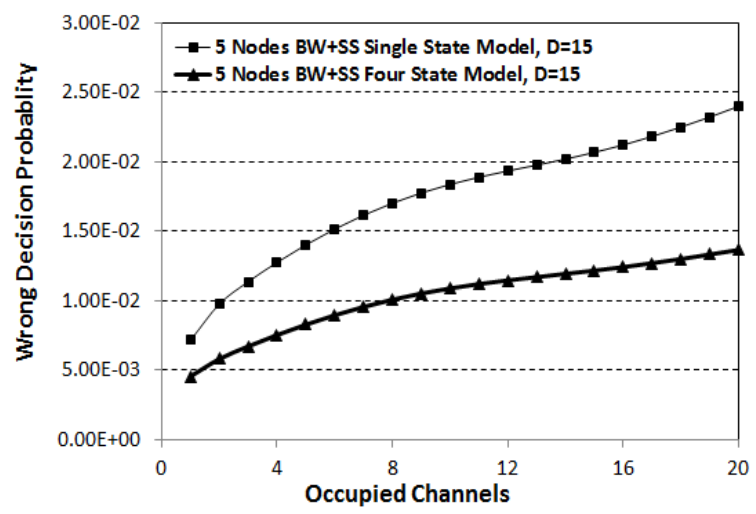

Fig 7: Wrong Decision Probability versus Traffic Density for BW plus RSS based SingleState and Four State Models with $\mathrm{D}=15 \mathrm{~ms}$

In single state model all the mobile nodes are considered in cooperative state, whereas in four state model all other states are also considered, the number of mobile nodes which are in 
cooperative state are in fraction in case of four state model. Hence the MHP, UHP and WDP are lesser in four state model. But this is only a formulation improvement to predict the probabilities accurately.

Fig. 8 shows improvement in probabilities versus bandwidth (traffic density) for four state model for different decision times, $\mathrm{D}=11$ to $15 \mathrm{~ms}$, considering $\mathrm{BW}$ plus RSS as performance metric. It can be noticed that there improvement in probability with increase in decision time and occupied number of channels

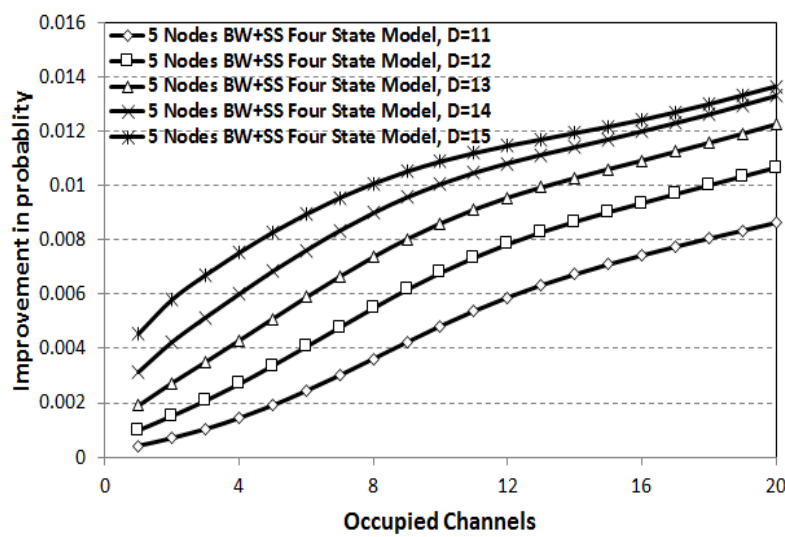

Fig 8: Improvement in Probability versus Traffic Density for BW plus RSS based Four State Models with for $D=11$ to $15 \mathrm{~ms}$

Fig. 9 shows Handover probabilities versus bandwidth (traffic density) for single state and four state model for decision time, $\mathrm{D}=11 \mathrm{~ms}$, considering BW plus RSS as performance metric. It can be noticed that there is reduction in handover probability when single state model is replaced with four state model and also Handover probability almost remains constant with increase in occupied number of channels.

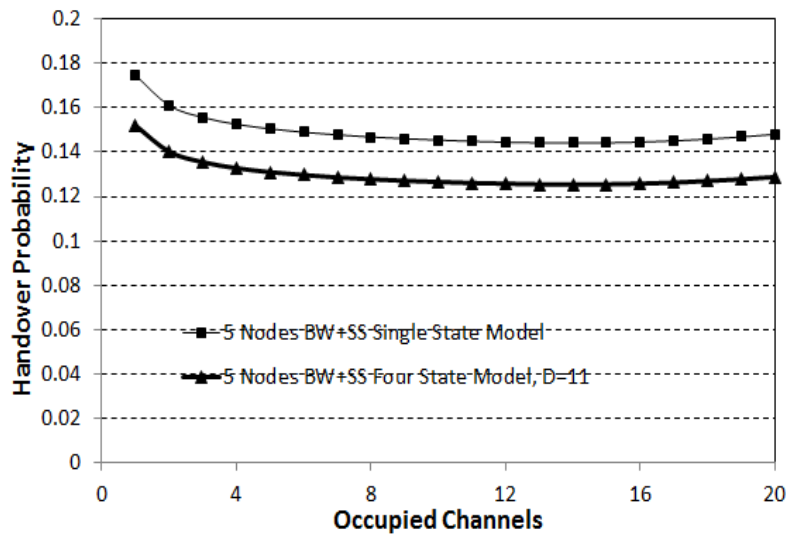

Fig 9: Handover Probability versus Traffic Density for BW plus RSS based SingleState and Four State Models with $D=11 \mathrm{~ms}$

\section{CONCLUSIONS}

In this work, handover probability, Unnecessary Handover Probability, Missing Handover Probability and Wrong Decision Probability are calculated for the bandwidth up to 20 in four state five node network model, considering BW and RSS as performance metric. The decision times are varied from $11 \mathrm{~ms}$ to $15 \mathrm{~ms}$. The four states of the model, where the mobile node can exist are cooperative, failed, selfish and malicious states. Considering only cooperative state, the model is referred to as single state model and is simplified model. Four state model on the other hand is very close to the actual scenario. It is proved with this investigation that, the probabilities of the UHP, MHP and WDP of four state model with BW plus RSS are much less than that of single state model as the number of mobile nodes which are in cooperative state are in fraction in case of four state model. Also it is observed that there is small amount of reduction in Handover probability in four state five node network model with BW plus RSS, as compared to single state model. UHP is less by at least $65 \%$ and MHP is less by at least $50 \%$ and WDP is less by $50 \%$. As future work this model can be further extended to models considering combination of additional parameters such as cost function, latency etc., along with RSS and BW so as to represent a case close to the actual scenarios and also this four state model can be extended to the work considering movement of the mobile node while in a vehicle.

\section{REFERENCES}

[1] S. Lee, K. Sriram, K. Kim, Y.H. Kim and N. Golmie, "Vertical handoff decision algorithms for providing optimized performance in heterogeneous wireless networks," IEEE Transactions on Vehicular Technology 58 (2) (2009) 865-881.

[2] B. R. Chandavarkar, G. Ram Mohan Reddy, "Survey Paper: Mobility Management in Heterogeneous Wireless Networks," International Conference on Communication Technology and System Design 2011, Procedia Engineering 30 (2012) 113 - 123, (c) 2011 Published by Elsevier Ltd.

[3] R. Berezdivin, R. Breinig and R. Topp, "Next-generation wireless communications concepts and technologies,' IEEE Comm. Mag. 40(3) (March 2002), pp. 108-116.

[4] A.K. Salkintzis, C. Fors and R. Pazhyannur, "WLANGPRS integration for next-generation mobile data networks," IEEE Wireless Comm. 9(5) (Oct. 2002), pp. $112-124$.

[5] I.F. Akyildiz, J. McNair, J. Ho, H. Uzunalioglu and W. Wang, "Mobility management in current and future communications networks," IEEE Network 12(4) (July/Aug. 1998), pp. 39-49.

[6] B. Liang and Z.J. Haas, "Predictive distance-based mobility management for multi-dimensional pcs networks," IEEE/ACM Trans- actions on Networking 11(5) (Oct. 2003), pp. 718-732

[7] Ram Kumar Singh, Amit Asthana, Akanksha Balyan, Shyam J. Gupta, Pradeep Kumar, "Vertical Handoffs in Fourth Generation Wireless Networks," International Journal of Soft Computing and Engineering (IJSCE), ISSN: 2231-2307, Vol. 2, Issue 2, May 2012.

[8] Sudipta Patowary, Nityananda Sarma and Siddhartha Sankar Satapathy "SINR based Vertical Handoff Algorithm between GPRS and Wi-Fi Networks," Special Issue of IJCCT, Vol.1 Issue 2, 3, 4; for International Conference [ACCTA-2010], 3-5 August 2010.

[9] Nilakshee Rajule, Prof. Bhavna Ambudkar, Dr. A. P Dhande, "Survey of vertical Handover Decision Algorithms," International Journal of Innovations in Engineering and Technology (IJIET).

[10] Xiaohuan Yany Y. Ahmet S_ekercio_gluy Sathya Narayanan "A Survey of Vertical Handover Decision 
Algorithms in Fourth Generation Heterogeneous Wireless Networks," Elsevier 2010.

[11] X. Cai, and C. Chi, "An Analytical Model for Performance Evaluation of Handover Decision Algorithms," in Proc. on 2nd International Conference on Communications and etworking, China, CHINACOM 2007, pp.1079-1083, 22-24 August 2007.

[12] Gabriele Tamea, Anna Maria Vegni, Tiziano Inzerilli, Roberto Cusani "A Probability based Vertical Handover Approach to Prevent Ping-Pong Effect," The Sixth International Symposium on Wireles Communication Systems 2009 (ISWCS09).

[13] Ben-Jye Chang, Shu- Lin, Ying-Hsin Liang, "Minimizing Roaming Overheads for Vertical Handoff in Heterogeneous Wireless Mobile Networks," Proceedings of the 2006 International Conference on Wireless Communications and Mobile Networks.

[14] C. Chi, X. Cai, R. Hao, and F. Liu, "Modeling and Analysis of Handover Algorithms," Proc. on IEEE Global Telecommunications Conference (GLOBECOM 2007), pp. 4473-4477, 26-30 November 2007, Washington, DC, USA.

[15] S. Akhila and Suthikshn Kumar, "Analysis of Handover Algorithms based on Wrong Decision Probability Model," International Journal of Wireless Networks and Communications, Volume 2, Number 3, pp. 165-173, 2010.

[16] S. Akhila and Suthikshn Kumar, "Reduction of Wrong Decisions for Vertical Handoff in Heterogeneous Wireless Networks," International Journal of Computer Applications (0975 - 8887) Volume 34- No.2, 2011.
[17] Akhila. S, Suthikshn Kumar, Sambasiva Rao 2012 "Study of multiple parameter algorithm for wrong decisions in vertical handovers in wireless heterogeneous networks," Elixir Network Engg., ISSN: 2229 - 712X.

[18] Suresh R. Halhalli, Subhash Kulkarni, K.S.R Anjaneyulu, S. Akhila, "Probability Modeling of Multi Node Wireless Networks," International Journal of Computer Applications (IJCA) (0975 - 8887) Volume 51-No.1, August 2012, pp. 22-27.

[19] Suresh R. Halhalli, Subhash Kulkarni, K.S.R Anjaneyulu, "Reduction of UHP in Heterogeneous Wireless Networks," International Conference for Convergence of Technology - 2014, 978-1-4799-37592/14/\$31.00@2014 IEEE.

[20] Patil Shweta and B.N. Manjunatha Reddy, "Modeling and Analysis of Two Node Network Model with Multiple states in Mobile Networks," International Journal of Computer Applications Technology and Research Volume 3-Issue 1, 52 - 55, 2014

[21] F. Xing and W. Wang: Modeling and Analysis of connectivity in Mobile Ad Hoc Networks with Misbehaving Nodes, IEEE ICC 2006 proceeding, IEEE Communications Society.

[22] Suresh R. Halhalli, Subhash Kulkarni, K.S.R Anjaneyulu, S. Akhila "Handover Modeling of Multiple States of Mobile Node in a Five Node Network Model," International Journal of Computer Applications (IJCA) (0975 - 8887) Volume 111- No.3, February 2015, pp 16-21 